\title{
Generic and patient-specific models of the arterial tree
}

\author{
Philippe Reymond · Orestis Vardoulis • \\ Nikos Stergiopulos
}

Received: 9 July 2012 / Accepted: 11 July 2012 / Published online: 29 July 2012

(C) Springer Science+Business Media, LLC 2012

\begin{abstract}
Recent advance in imaging modalities used frequently in clinical routine can provide description of the geometrical and hemodynamical properties of the arterial tree in great detail. The combination of such information with models of blood flow of the arterial tree can provide further information, such as details in pressure and flow waves or details in the local flow field. Such knowledge maybe be critical in understanding the development or state of arterial disease and can help clinicians perform better diagnosis or plan better treatments. In the present review, the state of the art of arterial tree models is presented, ranging from 0-D lumped models, 1-D wave propagation model to more complex 3-D fluid-structure interaction models. Our development of a generic and patient-specific model of the human arterial tree permitting to study pressure and flow waves propagation in patients is presented. The predicted pressure and flow waveforms are in good agreement with the in vivo measurements. We discuss the utility of these models in different clinical application and future development of interest.
\end{abstract}

Keywords Cerebral circulation - 1-D model - Wave propagation - Noninvasive measurements techniques . Heart model · Ventricular-vascular coupling · Blood flow simulation

P. Reymond $(\varangle) \cdot$ O. Vardoulis · N. Stergiopulos Laboratory of Hemodynamics and Cardiovascular Technology, EPFL / STI / IBI2 / LHTC, Ecole Polytechnique Fédérale de Lausanne, BM 5128, Station 17, 1015 Lausanne, Switzerland e-mail: philippe.reymond@a3.epfl.ch

\section{Introduction}

Blood flow phenomena and related hemodynamical forces play an important role in various forms of vascular disease. Pressure wave reflections, for example, play a pivotal role in the development of isolated systolic hypertension.

From a clinical standpoint, the assessment of hemodynamical forces within the systemic circulation is still difficult. For instance, in the cerebral circulation, pressure can be measured only invasively and flow, especially in small deep intracranial vessels such as those in the vicinity of the circle of Willis, cannot be measured directly with Doppler ultrasound. This renders modeling of blood flow within the cerebral circulation an attractive alternative.

Mathematical models of the arterial tree, when utilized in conjunction with in vivo data can lead to a better understanding of the physiopathology of the cardiovascular system. Modern imaging modalities such as MRI, CT and 3-D digital subtraction angiography provide high-definition images of the vessel geometry and thus can give precise description of the local 3-D geometry with millimeter resolution, a spatial definition which is necessary for accurate simulation of the flow field using appropriate computational fluid dynamic methodologies.

In this review article, we present briefly different approaches to modeling of the arterial tree, reporting the main advantages and disadvantages of each approach. We describe, with a bit more detail, our development of a model of the circulation which can be used to predict pressure and flow waves in generic or patient specific arterial trees. As an example, we show how the model is used to assess the effects of aortic graft implantation on aortic pressure waves. In the end, we discuss the utility of different arterial flow models in various clinical applications and foreseeable future developments of such models. 


\section{Models}

\subsection{Lumped parameter or 0-D models}

Lumped parameter models (often called also 0-D or Windkessel models) are models composed of in series and in parallel combinations of the resistive, compliant and inertial components of the arterial tree. The simplest and widely known lumped model of the arterial is the 2-element Windkessel model, first proposed by Otto Frank (1899). Lumped models are utilized to mimic parts or the entire arterial tree. They give information on flow and pressure; however they do not take into account wave propagation phenomena. Their advantage lies in the fact that only few global parameters are required to characterize the arterial network under consideration. Lumped parameter models have been used extensively in arterial modeling and research, with applications ranging from compliance estimation [1], to estimation of pressure and flow and to assessment of vascular-ventricular coupling [2].

\subsection{Distributed parameter or 1-D models}

1-D models result from the integration of Navier-Stokes equation over the arterial cross-section and are ideal for the study of pressure and flow wave propagation along the arterial tree. In the case of isolated systolic hypertension, the pressure load on the heart is affected by the contribution of pressure waves that travel forward and backward (reflected waves). The modeling of forward and reflected waves can be easily and accurately performed using appropriate 1-D models. In 1-D models, the main arteries of the arterial tree are decomposed into interconnected straight arterial segments. In each of these segments, one can either solve the integrated forms of the Navier-Stokes equations or use the oscillatory flow theory from $[3,4]$ or the electrical transmission line theory [5].

1-D models of the circulation have been extensively studied in the past to study cardiovascular physiology [511] and also extensively used to study pathologies, such as hypertension [12], arteriosclerosis [13], stenosis [6-8, 10, 14-16] and effect of anatomical variations of cerebral arteries and arterial occlusion [17].

\subsection{3-D models of the arterial tree}

Unlike the 1-D wave propagation models, 3-D models using computational fluid dynamics (CFD) approaches, are based on the numerical solution of the full Navier-Stokes equations. 3-D CFD models are appropriate to study complex flow structures in local geometries of interest, such as arterial bifurcations [18], the abdominal aorta [18], aneurysms [19] or close to cardiac valves, to mention only a few. 3-D models, often based on patient specific geometries, are increasingly used to analyze the local flow patterns at a specific point or location in the vasculature. The aim is to provide a better understanding of the flow fields and the resulting hemodynamical forces on the arterial wall, as these forces are implicated in the genesis, evolution and regression of vascular pathologies. The role of the wall shear stress on formation and stability of atherosclerotic plaques in the carotid bifurcation [20] and the growth and rupture of cerebral aneurysms [19] are two important examples that bolster this concept.

3-D models are still difficult to apply in extended regions of the arterial tree, because they are computationally intense and require detailed description of the 3-D geometry, a task which is difficult also to achieve over an extended part of the arterial tree. 3-D models require accurate boundary conditions, often expressed as velocity or pressure waveforms at the upstream and downstream boundaries of the computational domain. A multi-scale approach, where 3-D model is coupled to a distributed 1-D model is often utilized, where the 1-D model, serves to provide pressure and flow boundary conditions required by the 3-D solver [21].

Most of the 3-D models that are utilized to study blood flow consider the vessel wall to be a rigid structure. The basic underlying assumption is that the wall motion does not affect the flow field significantly. This assumption simplifies the numerical approach and is fully justifiable only in the cases where the wall motion is relatively small. However, when significant arterial wall deformation occurs during the heart cycle, as this is the case in flow around valves or in the human aorta, it becomes essential to consider deformation of the arterial wall. This is done by solving the solid mechanics problem for the deformation of the arterial wall. Numerous examples of a fluid-structure interaction approach (FSI) to solving blood flow phenomena in arteries can be found in the recent literature [22], however, methodologies are still under development. FSI modeling of a patient-specific vasculature is complex and computationally expensive due to the additional properties (arterial wall) and boundary conditions (outer surface of the arterial wall) needed for such simulation. However, the development of automated meshing processes in conjunction with the use of more efficient parallel algorithms may render the FSI approach applicable in research or clinical framework in the near future. For instance, this approach could be relevant to obtain blood flow details, like the temporal variation of wall shear stress, in regions that are prone to atherosclerotic plaques development [23] or development of cerebral aneurysms [19]. 


\section{Generic and patient-specific models}

A generic model is built on average arterial geometry and average arterial elastic properties representing, typically, a healthy person of a given age. This model can be used to simulate and study different aspects of blood flow physiology or pathology with scope to understand the phenomena themselves rather than applying the results to specific patient.

Interest in patient-specific models of the systemic circulation has increased in recent years [24-27]. A decrease in numerical simulations time in conjunction with increased availability of clinical data for geometry and elastic properties have made patient-specific models more feasible. For instance, patient-specific models are now utilized to assess the risk of aneurysm rupture, plan surgical procedures and predict treatment outcome. The reproducibility of patient-specific hemodynamic simulations has been reported in the literature for numerical simulation of cerebral aneurysms [28]. Such patient-specific approaches may lead to novel diagnostic and treatment planning tools in the future.

\section{Personal contribution to the field}

We have constructed a complete 1-D model of the arterial tree, which comprises 103 segments and includes a detailed description of all systemic arteries, including the coronaries, a detailed cerebral arterial tree, a heart model and appropriate description of wall viscoelasticity. The approach we followed was to compare the predictions of the generic 1-D model with the average pressure and flow waveforms measured noninvasively in a group of healthy young people (Fig. 1). The underlying hypothesis was that although the generic model would not represent precisely a specific individual it would represent quite well the "average" of the group.

The fluid mechanics equations were solved numerically to obtain pressure and flow throughout the arterial tree. The heart is modeled using the time varying elastance model, providing a versatile and physiologically relevant way to take into account parameters like heart rate, maximum contractility, and changes in preload and atrial pressure. The coronary arteries were modeled assuming a systolic flow impediment proportional to ventricular varying elastance. A nonlinear viscoelastic constitutive law for the arterial wall was considered while distal vessels were terminated with a three-element Windkessel model. We have included all main cerebral artery segments as well as all afferent and efferent arteries in the vicinity of the circle of Willis. This is useful when studying blood flow in cerebral aneurysm, as such circle is the most frequent location of cerebral aneurysms [29]. Many extracranial arteries, such as the superficial temporal arteries have also been added, in order to include points where pressure can be measured noninvasively and serve in the validation of the work.

Some data for pressure and flow in major systemic arteries are available, where Doppler measurements for flow velocity are accessible and tonometry (for pressure) is feasible [30]. However, non-invasive measurements of cerebral pressure and flow waves are scarce. Most of the measurements have been performed for extracranial arteries such as the common carotid by Doppler ultrasound [31] and the internal carotid and vertebral artery flow rate by MRI [32]. Few data are available for the efferent vessels of the circle of Willis (anterior, middle and posterior cerebral arteries [33]). For systemic arteries, there is no complete data set for pressure and flow measured at several arterial locations and where arterial properties are also measured or estimated on an individual basis. Hence, 1-D model validation cannot be based on data in the literature. Thus, we performed a more complete set of non-invasive measurements. Flow in the large arteries was quantified with PCMRI, cerebral flow detected with ultrasound Doppler and blood pressure measured with applanation tonometry. Figure 1 shows that the model is able to reproduce well the pressure and flow waveforms characteristics of an average subject.

On a second stage, we proceeded with the construction of a patient-specific model. Measurements of geometry, elasticity, flow and pressure were performed on the same subject. The model predictions were validated with noninvasive measurements of pressure and flow performed on the same person. Model predictions at different arterial locations compared well to measured flow and pressure waves at the same anatomical points (Fig. 2). The model predicted pressure and flow waveforms in good agreement with the in vivo measurements with regards to wave shape and features.

\subsection{Example of a specific application of an 1-D model to studying aortic grafts}

Clinicians and surgeons could also benefit from a robust tool that provides pre surgical overview on the potential outcome regarding central pressure and wave reflections after vascular angioplasty or total aortic replacement. The ability of the model to address surgical hemodynamic situations was shown with a study that focused on the impact of aortic grafts on central arterial pressures and wave reflections [36]. The Dacron material used for these grafts does not share the same mechanical properties with the native aortic tissue neither the same geometry. However, proximal aortic compliance and dimensions define the characteristic impedance of the arterial tree and the 


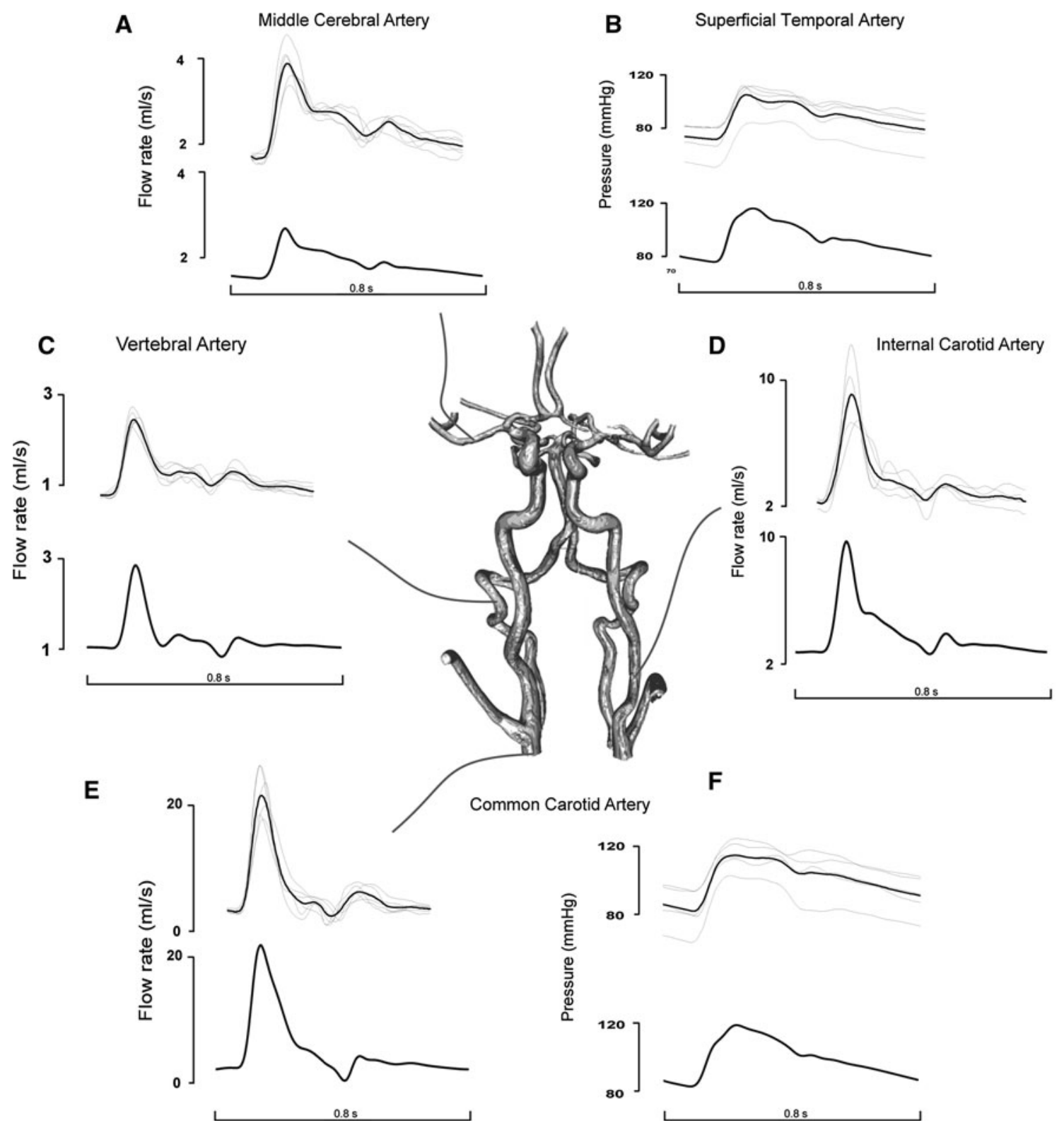

Fig. 1 Taken from [34]. Model results for a generic arterial tree (bottom panels) compared to in vivo measurements (top panels) at various cerebral artery locations. Thick line represents the averaged waveform. Blood flow was measured with color-coded duplex

ultrasound (a, c, d, e). Pressure was measured with applanation tonometry in the superficial temporal artery (b) and common carotid (f)

volumetric compliance of the ascending aorta stands for nearly $50 \%$ of total systemic compliance. Furthermore it is not clear yet how the implantation location may affect hemodynamics and whether a post-surgical increase in central pressure might be the result of increased impedance or augmented reflections. To address these questions the model was modified to incorporate a proximal aortic graft located at the aortic root and a distal aortic graft located at the descending thoracic aorta. Mechanical properties were derived by pressure diameter tests on a woven Dacron graft and a comparison with the data published by Langewouters

et al. [37] revealed an order of magnitude difference between aortic and graft compliance for average arterial tree pressures. Aortic systolic pressure and pulse pressure (PP) increased for both proximal and distal aortic grafts, with the increase being more pronounced in the case of a proximal graft. Proximal graft implantation resulted in $58 \%$ increase in the amplitude of the forward pressure wave. Inversely, for the distal graft the characteristic impedance remained unaffected and the PP increase was the result of amplified reflection waves generated by the compliance mismatch between the graft and the thoracic 


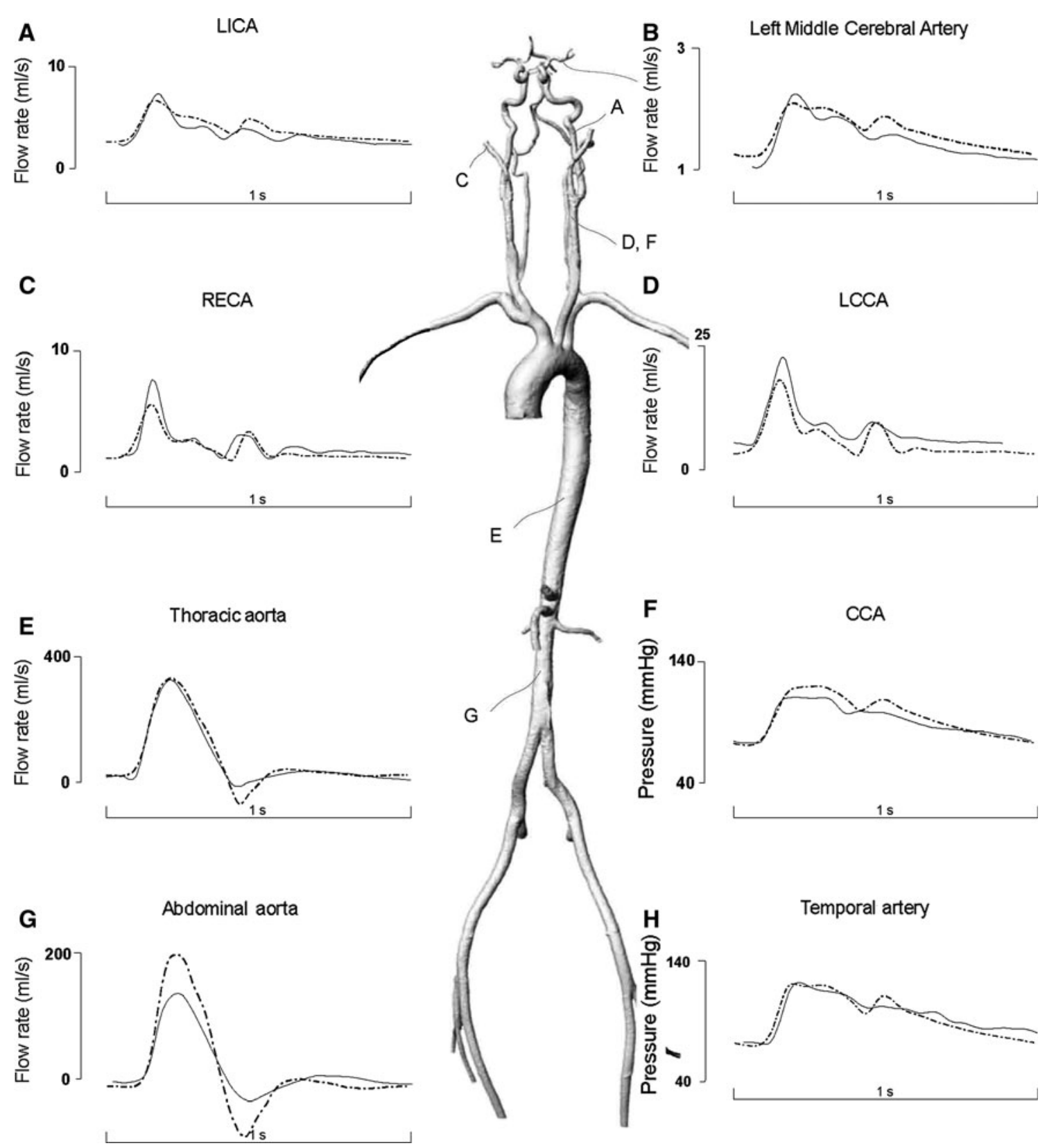

Fig. 2 Adapted from [35]. 1-D model predictions (dash dot) compared to in vivo measurements of flow and pressure waves (continuous line) for different systemic and cerebral arteries. Flow is measured with B-mode color-coded duplex flow imaging technique in the left internal carotid LICA (a), left middle cerebral artery LMCA (b) and right external carotid RECA (c), with PC-MRI in the left

aorta. The rise in PP is thus affected by both the position of the graft and the level of compliance mismatch. While studies for the in vivo confirmation are still undergoing, these result suggest that ascending aorta graft recipients are potentially at a greater risk for post-surgical systolic hypertension and therefore deserve closer blood pressure monitoring.

A number of limitations are present in our 1-D models. For instance, the autoregulation phenomenon, in response common carotid artery LCCA (d) in the thoracic aorta (e) and in the abdominal aorta (g). Pressure was measured with applanation tonometry in the common carotid artery CCA (f) and superficial temporal artery (h). The central picture is the representation of the 3-D geometry of the patient-specific arterial tree from MRI angiography

to hemodynamical stimuli, which is an important issue in cerebral blood flow mechanisms, was not considered and could be included in the future. Small distal vessels such as arterioles, capillaries, venules and veins were not modeled directly and may need to be investigated in detail. Addition of the venous tree and the pulmonary circulation could also permit to have a full circulation model, where extended physiopathological mechanisms including venous return or pulmonary flows could be studied. 


\section{Clinical applications and future prospects}

Coronary circulation can also be studied with 1-D models and can help understanding the physiological specificity of the coronary circulation [38], when contraction of the myocardium affects coronary perfusion. Many questions remain regarding the physiology and hemodynamics of coronary circulation and numerous models have been proposed. Classical models referred to the systolic extravascular model, waterfall model or intramyocardial pump model and the effect of the contraction can be affected by the elastance and/or pressure of the left ventricle. These different models can be studied with the help of a 1-D wave propagation approach. Pulmonary circulation can also be studied by the 1-D model to study, for instance, pulmonary hypertension. The interaction between arterial blood flow and cerebrospinal fluid can be also studied with coupled 1-D models of both systems. This interaction seems to play an important role in some cerebrospinal fluid-related pathologies [39].

Based on a person-specific in vivo measurements, a 1-D model can be used and optimized to find the better fit between measured and simulated pressure and flow waveforms, by adjusting key parameters such as compliance, geometry, and elastance [40]. Estimation of the arterial tree or heart model parameters can be useful to characterize the state of the arterial tree.

Central aortic pressure is a better indicator of cardiac risk than distal peripheral pressure. However, aortic pressure is only available with invasive measurements. Thus, the use of a transfer function between peripheral and central pressure could be a useful clinical tool to help for a better understanding of the cardiac risk in a non-invasive manner. A 1-D wave propagation model is well suited for this task [41, 42].

1-D model has also been used recently to evaluate the wave propagation theory of a method that measures the stiffness of central arteries with a new and non invasive device [43].

In the case of isolated systolic hypertension, pressure load on the heart is affected by the contribution of pressure waves that travel forward and backward (reflected waves) and affect central pulse pressure. In this situation, hemodynamics is governed by global wave phenomena and to a lesser extent by local 3-D flow. Therefore, wave propagation models (1-D models) are the most appropriate.

Arterial tree model may be also utilized to generate large pressure waveform datasets by altering model parameters within physiological range. In studies were processing of various pressure waveforms is required to test methods for the estimation of cardiac output [44] or for the estimation of total systemic compliance by PWV [45] the arterial tree model can play a pivotal role in reducing the need for in vivo data while in the validation zone.

1-D models are also able to provide pressure and flow waveforms in an extended region of the arterial tree, which is useful for diagnostics, treatment planning [27] and for surgical planning [46]. Outcome of bypass surgery predicted by a 1-D wave propagation model with MRI data has been reported [47]. Pulse wave propagation models can be applied to predict the hemodynamic of an arteriovenous fistula in hemodialysis [48].

\section{Conclusion}

This review presents different human arterial tree models that have been used in the past or are in development. We presented the development of a generic arterial tree that predicted pressure and flow at systemic circulation locations with a good agreement as compared to in vivo measurements. A person-specific model was also presented, predicting pressure and flow waveforms with very good accuracy. Patient-specific models have potential to be used in a clinical environment and could be useful for providing better diagnosis or even better treatment planning in the near future.

Acknowledgments This work was supported by the by the European Commission contract no. IST-027703@neurIST Project and by the Center for Biomedical Imaging (CIBM) of the Geneva-Lausanne Universities, the EPFL and the University Hospitals of Geneva and Lausanne. Special thanks go to Prof. Jean-Claude Chevrolet for his clinical expertise.

\section{References}

1. Stergiopulos N, Meister JJ, Westerhof N. Simple and accurate way for estimating total and segmental arterial compliance: the pulse pressure method. Ann Biomed Eng. 1994;22(4):392-7.

2. Fitchett DH. LV-arterial coupling: interactive model to predict effect of wave reflections on LV energetics. Am J Physiol. 1991;261(4 Pt 2):H1026-33.

3. Witzig K. Über erzwungene Wellenbewegungen zäher, inkommpressibler Flüssigkeiten in elastischen Röhren. Bern; 1914.

4. Womersley JR. Method for the calculation of velocity, rate of flow and viscous drag in arteries when the pressure gradient is known. J Physiol. 1955;127(3):553-63.

5. Westerhof N, Bosman F, De Vries CJ, Noordergraaf A. Analog studies of the human systemic arterial tree. J Biomech. 1969;2(2):121-43

6. Avolio AP. Multi-branched model of the human arterial system. Med Biol Eng Comput. 1980;18(6):709-18.

7. Cassot F, Zagzoule M, Marc-Vergnes JP. Hemodynamic role of the circle of Willis in stenoses of internal carotid arteries. An analytical solution of a linear model. J Biomech. 2000;33(4): 395-405.

8. Meister J-J. Mesure par échographie Doppler et modélisation théorique de l'effet de troubles cardiaques sur la pression et le débit artériels. Lausanne; 1983. 
9. Schaaf BW, Abbrecht PH. Digital computer simulation of human systemic arterial pulse wave transmission: a nonlinear model. J Biomech. 1972;5(4):345-64.

10. Stergiopulos N, Young DF, Rogge TR. Computer simulation of arterial flow with applications to arterial and aortic stenoses. J Biomech. 1992;25(12):1477-88.

11. Zagzoule M, Marc-Vergnes JP. A global mathematical model of the cerebral circulation in man. J Biomech. 1986;19(12): 1015-22.

12. Westerhof BE, van den Wijngaard JP, Murgo JP, Westerhof N. Location of a reflection site is elusive: consequences for the calculation of aortic pulse wave velocity. Hypertension. 2008; 52(3):478-83. doi:10.1161/HYPERTENSIONAHA.108.116525.

13. Raines JK, Jaffrin MY, Shapiro AH. A computer simulation of arterial dynamics in the human leg. J Biomech. 1974;7(1):77-91.

14. Kufahl RH, Clark ME. A circle of Willis simulation using distensible vessels and pulsatile flow. J Biomech Eng. 1985; 107(2):112-22.

15. Stettler JC, Niederer P, Anliker M. Theoretical analysis of arterial hemodynamics including the influence of bifurcations. Part I: mathematical models and prediction of normal pulse patterns. Ann Biomed Eng. 1981;9(2):145-64.

16. Stettler JC, Niederer P, Anliker M, Casty M. Theoretical analysis of arterial hemodynamics including the influence of bifurcations. Part II: critical evaluation of theoretical model and comparison with noninvasive measurements of flow patterns in normal and pathological cases. Ann Biomed Eng. 1981;9(2):165-75.

17. Alastruey J, Parker KH, Peiro J, Byrd SM, Sherwin SJ. Modelling the circle of Willis to assess the effects of anatomical variations and occlusions on cerebral flows. J Biomech. 2007;40(8): 1794-805.

18. Taylor CA, Hughes TJR, Zarins CK. Finite element modeling of blood flow in arteries. Comput Methods Appl Mech Eng. 1998;158(1-2):155-96.

19. Cebral JR, Castro MA, Burgess JE, Pergolizzi RS, Sheridan MJ, Putman CM. Characterization of cerebral aneurysms for assessing risk of rupture by using patient-specific computational hemodynamics models. AJNR. 2005;26(10):2550-9.

20. Steinman DA. Image-based computational fluid dynamics modeling in realistic arterial geometries. Ann Biomed Eng. 2002; 30(4):483-97.

21. Marzo A, Singh P, Larrabide I, Radaelli A, Coley S, Gwilliam M, Wilkinson ID, Lawford P, Reymond P, Patel U, Frangi A, Hose DR. Computational hemodynamics in cerebral aneurysms: the effects of modeled versus measured boundary conditions. Ann Biomed Eng. 2011;39(2):884-96. doi:10.1007/s10439-010-0187-z.

22. Crosetto P, Reymond P, Deparis S, Kontaxakis D, Stergiopulos N, Quarteroni A. Fluid structure interaction simulation of aortic blood flow. Comput Fluids. 2011;43:46-57. doi:10.1016/ j.compfluid.2010.11.032.

23. Cheng C, Tempel D, van Haperen R, van der Baan A, Grosveld F, Daemen MJ, Krams R, de Crom R. Atherosclerotic lesion size and vulnerability are determined by patterns of fluid shear stress. Circulation. 2006;113(23):2744-53. doi:10.1161/CIRCULATIONAHA. 105.590018.

24. Gerbeau JF, Chapelle D. Numerical simulation of the cardiovascular system. Med Sci (Paris). 2005;21(5):530-4.

25. Perktold K, Rappitsch G. Computer simulation of local blood flow and vessel mechanics in a compliant carotid artery bifurcation model. J Biomech. 1995;28(7):845-56.

26. Quarteroni A, Formaggia L, Veneziani A. Cardiovascular Mathematics Modeling and simulation of the circulatory system. Milan: Springer; 2009.

27. Taylor CA, Figueroa CA. Patient-specific modeling of cardiovascular mechanics. Annu Rev Biomed Eng. 2009;11:109-34.
28. Radaelli AG, Augsburger L, Cebral JR, Ohta M, Rufenacht DA, Balossino R, Benndorf G, Hose DR, Marzo A, Metcalfe R, Mortier P, Mut F, Reymond P, Socci L, Verhegghe B, Frangi AF. Reproducibility of haemodynamical simulations in a subjectspecific stented aneurysm model-a report on the Virtual Intracranial Stenting Challenge 2007. J Biomech. 2008;41(10): 2069-81. doi:10.1016/j.jbiomech.2008.04.035.

29. Yasargil MG. Microneurosurgery. Microsurgical anatomy of the basal cisterns and vessels of the brain, diagnostic studies, general operative techniques and pathological considerations of the intracranial aneurysms, vol I. New York: Thieme Medical; 1984.

30. Kelly R, Hayward C, Avolio A, O'Rourke M. Noninvasive determination of age-related changes in the human arterial pulse. Circulation. 1989;80(6):1652-9.

31. Holdsworth DW, Norley CJ, Frayne R, Steinman DA, Rutt BK. Characterization of common carotid artery blood-flow waveforms in normal human subjects. Physiol Meas. 1999;20(3):219-40.

32. Ford MD, Alperin N, Lee SH, Holdsworth DW, Steinman DA. Characterization of volumetric flow rate waveforms in the normal internal carotid and vertebral arteries. Physiol Meas. 2005;26(4): 477-88.

33. Aaslid R, Lash SR, Bardy GH, Gild WH, Newell DW. Dynamic pressure-flow velocity relationships in the human cerebral circulation. Stroke J Cereb Circ. 2003;34(7):1645-9.

34. Reymond P, Merenda F, Perren F, Rufenacht D, Stergiopulos N. Validation of a one-dimensional model of the systemic arterial tree. Am J Physiol Heart Circ Physiol. 2009;297(1):H208-22. doi:10.1152/ajpheart.00037.2009.

35. Reymond P, Bohraus Y, Perren F, Lazeyras F, Stergiopulos N. Validation of a patient-specific one-dimensional model of the systemic arterial tree. Am J Physiol Heart Circ Physiol. 2011;301(3):H1173-82. doi:10.1152/ajpheart.00821.2010.

36. Vardoulis O, Coppens E, Martin B, Reymond P, Tozzi P, Stergiopulos N. Impact of aortic grafts on arterial pressure: a computational fluid dynamics study. Eur J Vasc Endovasc Surg. 2011;42(5):704-10. doi:10.1016/j.ejvs.2011.08.006.

37. Langewouters GJ. Visco-elasticity of the human aorta in vitro in relation to pressure and age. 1982.

38. Rogers PA, Kiyooka T, Chilian WM. Is there a need for another model on the pulsatile nature of coronary blood flow? Am J Physiol Heart Circ Physiol. 2006;291(3):H1034-5.

39. Martin BA, Reymond P, Novy J, Baledent O, Stergiopulos N. A coupled hydrodynamic model of the cardiovascular and cerebrospinal fluid system. Am J Physiol Heart Circ Physiol. 2012;302(7):H1492-509. doi:10.1152/ajpheart.00658.2011.

40. Leguy CA, Bosboom EM, Gelderblom H, Hoeks AP, van de Vosse FN. Estimation of distributed arterial mechanical properties using a wave propagation model in a reverse way. Med Eng Phys. 2010;32(9):957-67. doi:10.1016/j.medengphy.2010.06.010.

41. Stergiopulos N, Westerhof BE, Westerhof N. Physical basis of pressure transfer from periphery to aorta: a model-based study. Am J Physiol. 1998;274(4 Pt 2):H1386-92.

42. Westerhof BE, Guelen I, Stok WJ, Wesseling KH, Spaan JA, Westerhof N, Bos WJ, Stergiopulos N. Arterial pressure transfer characteristics: effects of travel time. Am J Physiol Heart Circ Physiol. 2007;292(2):H800-7. doi:10.1152/ajpheart.00443.2006.

43. Trachet B, Reymond P, Kips J, Swillens A, De Buyzere M, Suys B, Stergiopulos N, Segers P. Numerical validation of a new method to assess aortic pulse wave velocity from a single recording of a brachial artery waveform with an occluding cuff. Ann Biomed Eng. 2010;38(3):876-88. doi:10.1007/s10439-010-9945-1.

44. Papaioannou TG, Vardoulis O, Stergiopulos N. The "systolic volume balance" method for the noninvasive estimation of cardiac output based on pressure wave analysis. Am J Physiol Heart Circ Physiol. 2012;302(10):H2064-73. doi:10.1152/ajpheart.00052.2012. 
45. Vardoulis O, Papaioannou TG, Stergiopulos N. On the estimation of total arterial compliance from aortic pulse wave velocity. Ann Biomed Eng. 2012;. doi:10.1007/s10439-012-0600-x.

46. Wan J, Steele B, Spicer SA, Strohband S, Feijoo GR, Hughes TJ, Taylor CA. A one-dimensional finite element method for simulation-based medical planning for cardiovascular disease. Comput Methods Biomech Biomed Eng. 2002;5(3):195-206.
47. van de Vosse FN, Stergiopulos N. Pulsewave propagation in the arterial tree. Annu Rev Fluid Mech. 2011;43:467-99.

48. Huberts W, Bode AS, Kroon W, Planken RN, Tordoir JH, van de Vosse FN, Bosboom EM. A pulse wave propagation model to support decision-making in vascular access planning in the clinic. Med Eng Phys. 2012;34(2):233-48. doi:10.1016/j.medengphy. 2011.07.015. 